\title{
INTERVENCIONES ENFERMERAS EN CUIDADOS SANITARIOS DE SEXUALIDAD PARA MUJERES CON LESIÓN MEDULAR LUMBAR EN CATALUÑA
}

\author{
Esther Sánchez Raja \\ Presidenta de la Asociación Nacional de Salud Sexual y Discapacidad (ANSSYD) \\ esanchezra@gmail.com \\ Patricia López Lara \\ Colaboradora de la Asociación Nacional de Salud Sexual y Discapacidad (ANSSYD) \\ patricialopezlara@gmail.com \\ María Honrubia Pérez \\ Vicepresidenta de la Asociación Nacional de Salud Sexual y Discapacidad (ANSSYD) \\ mhonrubia@ub.edu
}

Fecha de Recepción: 1 Marzo 2018

Fecha de Admisión: 10 Abril 2018

\section{RESUMEN}

Este plan de promoción de la salud se centra en una temática específica, la intervención enfermera en sexualidad tras una lesión medular lumbar, en un género concreto, la mujer y una población específica, la catalana. La enfermería como profesión sanitaria, somos agentes de salud, y actuamos en torno a ella, además somos el nexo de unión perfecto entre el equipo médico y el social. Nuestra formación sanitaria en cuanto a promoción, prevención e intervenciones en salud, garantiza el correcto fomento de la autonomía y la independencia mediante los planes de educación para la salud. Referencias teóricas en enfermería tomadas en cuenta destaca la teoría de la valoración de los procesos funcionales de Margorie Gordon, quien otorga un patrón funcional exclusivo a la sexualidad y la reproducción. Metodología: el desarrollo del proyecto es viable mediante el modelo Precede. A través de intervenciones en los ámbitos de conocimientos, habilidades y actitudes, orientadas en su conjunto a modificar la conducta sexual y resultar en una vida sexual satisfactoria en armonía con su diversidad funcional.

Palabras clave: sexualidad; salud sexual; lesión medular; discapacidad; diversidad funcional; plan de promoción de la salud; enfermería; intervenciones; precede

\section{ABSTRACT}

Nursing interventions in sexuality health care for women with lumbar medicine injury in Catalonia: health promotion plan.

This health promotion plan focuses on a specific theme, the nurse intervention in sexuality after a spinal cord injury, in a specific gender, the woman and a specific population, the Catalan one. 


\section{INTERVENCIONES ENFERMERAS EN CUIDADOS SANITARIOS DE SEXUALIDAD PARA MUJERES CON LESIÓN MEDULAR LUMBAR EN CATALUÑA}

Nursing as a health profession, we are health agents, and act around it, we are also the perfect link between the medical team and the social. Our health training in terms of promotion, prevention and health interventions guarantees the correct promotion of autonomy and independence through education plans for health. Theoretical references in nursing taken into account highlights the theory of the evaluation of the functional processes of Margorie Gordon, who grants an exclusive functional pattern to sexuality and reproduction. Methodology: the development of the project is viable through the Precede model. Through interventions in the fields of knowledge, skills and attitudes, oriented as a whole to modify sexual behavior and result in a satisfactory sexual life in harmony with its functional diversity.

Keywords: sexuality; sexual health; spinal cord injury; disability; functional diversity; health promotion plan; nursing; interventions; precede

\section{INTRODUCCION}

Existen dos etiologías de lesión medular, la congénita y la adquirida, ya sea debida a un traumatismo 0 a una enfermedad degenerativa ${ }^{i}$. En este proyecto se tendrán en cuenta únicamente las adquiridas, a nivel lumbar y en concreto aquellas sufridas por mujeres.

Las mujeres afectadas por este tipo de lesiones plantean una problemática en cuanto a la vivencia de la sexualidad. Se encuentran en una situación en la que la necesidad de información y formación ha de estar cubierta. Como profesión enfermera, profesional sanitario y agentes de salud, estamos obligados a dar respuesta a esta problemática. .i $^{\text {i }}$

El presente proyecto se basa en la Valoración de enfermería aportada por Margorie Gordon (1987), en cuanto a los patrones funcionales, la cual especifica un único patrón para la Sexualidad y la Reproducción, el cual permite desarrollar los diagnósticos de enfermería NANDA. En el modelo social basado en derechos y el marco legislativo actual. El método utilizado para el desarrollo del plan de promoción de la salud, es el Precede.

"La sexualidad va más allá, es una dimensión que se mantiene desde el instante de la concepción hasta la muerte" (Fallas, 2009).

El proyecto, es cohesionar las necesidades y ofrecer un abordaje holístico de la sexualidad de las mujeres con lesión medular, partiendo de la base del aprendizaje, para volver a enseñar como poder disfrutar de su sexualidad y en el caso que así lo deseen tener relaciones sexuales satisfactorias y placenteras, con la formación realizada desde las consultas de enfermería podemos colaborar y ayudar a conseguir la independencia de la mujer como proveedora y receptora de placer, aumentar su autoestima, empoderarla a través de la sexualidad, al margen de la capacidad reproductiva.

Es necesario aclarar el concepto de diversidad funcional, en contra del de discapacidad, para hacer referencia a la discapacidad física, promovido desde el Foro de Vida Independiente en 2004, quienes reivindican que la discapacidad la otorga la sociedad, con sus presiones y limitaciones y no aquellas personas que por medio de una lesión física precisan de ayudas técnicas.

\section{SEXUALIDAD Y LESIÓN MEDULAR}

La patología asociada a la mujer lesionada medular (LM), se produce una disminución de la capacidad de lubricación vaginal y concienciación orgásmica, la dificultad para llegar al orgasmo es mayor si la lesión es completa y se sitúa por encima de L2. La fertilidad y el deseo se mantienen intactos. Tras el periodo agudo de la lesión transcurre un periodo de amenorrea transitoria de entre 0 y 6 ciclos. Becker, E. (1978); Oliver,

(2011), Curcoll M.L, Vidal J. (1992)

La LM, supone trastorno emocional severo, lógico en una situación nueva y traumática, inesperada e incontrolable para la persona que lo sufre y para su entorno más cercano. 
Tanto si se tiene pareja en el momento de la lesión como si no supone un reto de adaptación social y de rol del ser humano. Existe una disminución de la auto percepción como ser sexuado. La pérdida de sensibilidad provoca un cambio en la vivencia de la sexualidad.

La percepción propia se ve alterada, las sensaciones y la movilidad, suponen un obstáculo a la hora de relacionarse, de mantener relaciones sexuales. Esta se puede ver alterada por el cambio de imagen, los mitos y las falsas creencias, las características de la personalidad previa a la LM son determinantes para la futura rehabilitación y la capacidad de adaptación. El temor a no resultar sexualmente atractivo/a, la dependencia de terceros, la dificultad a la hora de vestirse o desvestirse, la menor habilidad de adoptar posturas cómodas y satisfactorias... (Hammond y Burns, 2000)

Previo al abordaje técnico propiamente dicho es importante trabajar el aspecto emocional y de capacidad, informar y educar en cuanto a las expectativas reales de sensación en el coito y desmitificar tabúes.

La diferencia entre el hombre y la mujer es evidente, la mujer parte de un modelo de sexualidad menos genitalizado que el hombre, basado en la comunicación, la ternura, las caricias y el juego erótico, etc; y debe aprovecharlo para disfrutar de su nuevo modelo de sexualidad. (Sánchez Ramos y De Pinto, 2000)

\section{JUSTIFICACIÓN}

La justificación del presente proyecto es viable dentro del marco legal actual y del Modelo Social basado en Derechos.

Se aplica con la ley 51/2003 Igualdad de oportunidades y no discriminación en la accesibilidad de las personas con discapacidad.

Tras la Convención de las Naciones Unidas Sobre los Derechos de las Personas con Discapacidad ,2006 (CDPD) donde se equiparan los derechos de las personas con discapacidad a las del común de la población. Se exige la adopción de medidas para solventar estas carencias tales como:

Disminuir las actitudes negativas como los obstáculos a la rehabilitación y la carencia de profesionales especializados.

Fomentar la sensibilización pública y comprensión de la discapacidad.

Asegurar los recursos de formación a instituciones docentes.

España se acoge a dicha normativa de Derechos en 2008 y se materializan en la Ley 26/2011 de adaptación normativa de la CDPD por la cual se impulsan cambios normativos y de plazos, necesarios para el complimiento de los compromisos adquiridos en dicha convención.

La Ley 3/2010 de Salud Sexual y Reproducción asistida, recoge en uno de sus artículos el derecho de las personas con discapacidad al acceso y los recursos para disfrutar de una salud sexual y reproductiva libre, sin limitaciones.

La intervención en enfermería la respalda la Ley Orgánica de Salud Sexual y Reproductiva e Interrupción voluntaria del embarazo de 2010 de obligatorio cumplimiento, mediante la cual se exige al personal sanitario y educativo dar respuesta a las demandas de información y formación en el campo de la sexualidad y en especial a las personas con vulnerabilidad.Esta ley no ha sido desarroIlada, por lo que no hay reglamento al respecto, los recursos asociados a esta no están aprobados, por lo que las subvenciones necesarias son inexistentes. Esto genera una problemática en cuanto a limitaciones de acceso las necesidades sociales y educacionales no están cubiertas, por la falta de oferta al respecto.

El referente bioético en el que se basa dicho proyecto se respalda en la Declaración Universal sobre Bioética y Derechos Humanos, de la Unesco, 2005 referente junto a la CDPD, ONU, 2006. 


\section{INTERVENCIONES ENFERMERAS EN CUIDADOS SANITARIOS DE SEXUALIDAD PARA MUJERES CON LESIÓN MEDULAR LUMBAR EN CATALUÑA}

Asistencialmente los profesionales pueden acogerse el Código de Buenas Prácticas para Profesionales en Sexualidad y Diversidad Funcional (2015) redactado por la Asociación Nacional de Salud Sexual y Discapacidad ANSSYD y Sex Asistent, en colaboración con la" Fundació La Caixa".

\section{ANTECEDENTES}

La sexualidad y el sexo se siguen considerando tabú en la sociedad actual, si mas no en el concepto de sexo asociado a la mujer, únicamente guiado por la capacidad reproductiva, la sumisión y la dependencia. En el caso de la mujer lesionada medular, no es diferente, se acentúan aún más si cabe. Los estudios realizados y las terapias efectuadas, por médicos, ginecólogos, sexólogos y psicólogos, tras la lesión en el campo de la sexualidad y en concreto en mujeres, son, casi inexistentes. Todos tenían como hilo conductor la reproducción tras la lesión. La sexualidad de la mujer y la capacidad de dar y recibir placer quedaban en un segundo plano, se priorizaba más en cuanto a aspectos de calidad de vida (CV) diaria si considerar a la sexualidad como un item.importante a tener en cuenta

Tras la primera reunión anual de la Sociedad Medica de Paraplejia (Atenas 1982-1989) se creía que solo el hombre veía afectada su capacidad sexual, en cuanto a funcionalidad, y se aseguraba que durante la relación sexual, la mujer es el miembro de la pareja más pasivo de los dos, ella recibe mientras que el hombre da, por lo tanto es lógico concluir que el acto no afecta a la mujer tanto como al hombre (Turk, 1982). Desmentido afortunadamente por Lowe, M. (1983) y Carole, S. (1984) quienes demuestran que no hay disminución del impulso emocional después de una lesión medular, tanto en el hombre como en la mujer.

Anteriormente se había publicado el libro de Female sexuality following spinal cord injury (Becker, E. 1978), en el que desde su propia perspectiva, afectada por una lesión medular, describe los aspectos prácticos de las relaciones sexuales en mujeres con lesión medular. En el libro plasma los resultados de entrevistas realizadas a mujeres con lesión medular, haciendo hincapié en los detalles asociados a los sentimientos y la adaptación psicológica de las mujeres.

En España, los estudios con mayor repercusión es este campo vienen dados por la Fundación Guttmann. Institución dedicada a la rehabilitación de personas con lesiones medulares. El inicio del programa de atención en sexualidad inició en 1977, tras la demanda de los pacientes, y necesidades de respuestas sobre su vida sexual después de la lesión. En aquel entonces el abordaje era básicamente médico-funcional. Posteriormente fueron adaptando las intervenciones y formándose en el campo para ofrecer soluciones y asesoramiento eficaz en sexualidad. En su estudio "Sexualidad de la mujer lesionada medular" (Curcoll, M.L, Vidal, J.1989) se describen y se diferencian los conceptos de erotismo, relación y procreación. Los resultados evidencian una total correlación entre la riqueza de las fantasías y la plenitud de la vida sexual en las mujeres lesionadas.

En la actualidad existen gran cantidad de estudios, publicados en revistas especializadas en medicina, psicología y sociología, que recogen artículos relacionados con la sexualidad y la discapacidad. Cabe destacar el trabajo de revistas especializadas dedicadas a la discapacidad y sexualidad, como Sexuality and Disability. En España las referencias más relevantes en cuanto a bibliografía al respecto vienen dadas por autores como Béjar y Béjar (1989), Curcoll y Vidal (1992) y De Pinto y Sánchez (2000).

En el caso de la enfermería la publicación SCI Nursing: A publication of the American Association of Spinal Cord Injury Nurses, donde se aborda el tema de la sexualidad y la lesión medular. El principal motor en cuanto a información y formación viene dado por los recursos aportados por las Asociaciones de personas con discapacidad, como ANSSYD, CERMI, ONCE, Dones no Estándar, entre otras. A nivel asistencial los principales organismos referentes en este campo son el Hospital de Parapléjicos de Toledo y el Instituto Neurorrehabilitador Gutmann de Badalona. 


\section{OBJETIVOS:}

\section{Objetivo Principal}

Mejorar la salud sexual de las mujeres tras una lesión medular lumbar mediante la implantación de un programa de promoción de la salud.

\section{Objetivos específicos:}

Aumentar el conocimiento en salud sexual de las mujeres con lesión medular lumbar y su entorno

Mejorar el auto concepto y la autoexploración de las mujeres con lesión medular lumbar

Incrementar la participación de la pareja 0 entorno en la salud sexual de la mujer con lesión medular.

\section{SUJETOS DE ESTUDIO}

El plan de promoción va dirigido a mujeres de la comunidad autónoma de Cataluña. De edades comprendidas entre los 18 y los 50 años. Que sufran una lesión medular lumbar parcial 0 total, no congénita, de al menos 2 años de evolución, pasado ya el período agudo de la lesión. Estas deben haber experimentado relaciones sexuales previas a la lesión, satisfactorias o no, sin necesidad de haber experimentado el orgasmo. No tienen por qué tener pareja, pero, tienen la necesidad de reeducar y adaptar su actividad sexual tras la lesión.

Las mujeres podrán conocer el plan de promoción a través de asociaciones de apoyo y soporte a personas con discapacidad y/0 de diversidad funcional y sexualidad, que promuevan cursos $y$ actividades para mejorar la calidad de vida de las personas lesionadas y sus familias.

El proceso de captación se da mediante la facilitación de información detallada y publicidad de reclamo (poster y tríptico) en Atención Primaria y en las asociaciones anteriormente mencionadas en el que se explique el tema a tratar y los beneficios de las participantes. Las mujeres que estén en contacto con las asociaciones y tengan la necesidad de aumentar la satisfacción de sus relaciones sexuales, podrán entrar en el proceso de selección.

El proceso de selección viene dado al incluir y excluir según los criterios de inclusión/ exclusión detallados a continuación. (Tabla 1)

Tabla 1 Criterios de inclusión /exclusión

\begin{tabular}{|l|l|}
\hline Criterios de inclusión & Criterios de exclusión \\
\hline Mujer & Hombre \\
\hline Edad 18-50años & Menores o postmenopáusicas. \\
\hline LM lumbar, no congénita parcial o total & Otro tipo de LM o de origen congénito \\
\hline $\begin{array}{l}\text { Intervención pasado el periodo agudo de } \\
\text { la lesión (2 años) }\end{array}$ & $\begin{array}{l}\text { Intervención durante el periodo agudo de } \\
\text { la lesión. }\end{array}$ \\
\hline Experiencias sexuales previas & Sin experiencias sexuales previas \\
\hline Con pareja estable o soltera. & \\
\hline $\begin{array}{l}\text { Con necesidad de aumentar la satisfacción } \\
\text { sexual. }\end{array}$ & \\
\hline $\begin{array}{l}\text { Con dificultad para mantener relaciones } \\
\text { sexuales post. LM }\end{array}$ & \\
\hline $\begin{array}{l}\text { Con disposición para mejorar su situación } \\
\text { actual, de no satisfacción sexual. }\end{array}$ & \\
\hline
\end{tabular}




\section{INTERVENCIONES ENFERMERAS EN CUIDADOS SANITARIOS DE SEXUALIDAD PARA MUJERES CON LESIÓN MEDULAR LUMBAR EN CATALUÑA}

\section{METODOLOGIA:}

Los progresos de la ciencia y la tecnología requieren cada vez más para la profesión de enfermería, una preparación acorde a los nuevos estilos de salud, con el fin de desarrollar con eficacia y eficiencia los cuidados que ofrece al usuario. Los profesionales de enfermería tienen el compromiso de usar las capacidades en la actualización de conocimientos teórico-prácticos que les permitan la direccionalidad de su práctica profesional. La atención a la discapacidad no siempre ha estado ligada al concepto de cuidados de enfermería, sin embargo las enfermeras/os están capacitadas/os para proporcionar atención y satisfacer las complejas necesidades físicas y psicosociales de las personas con LM y sus familias (Sánchez 2015).

La enfermería comunitaria es la rama de la enfermería dedicada a la prevención y promoción de la salud. Desde esa especialidad se asume la competencia como colectivo orientador y el nexo de unión perfecto entre el equipo de salud y el paciente. En España se recoge la especialidad de enfermería comunitaria en el BOE Orden SAS/1729/2010, de 17 de junio, por la que se aprueba y publica el programa formativo de la especialidad de Enfermería Familiar y Comunitaria.

En la actualidad la valoración de enfermería más indicada para trabajar en Sexualidad, para desarrollar los diagnósticos enfermeros NANDA, intervenciones y actividades NIC y los criterios de resultados NOC dentro de un plan de PdIS, es la que describe los Patrones Funcionales de Gordon, M (1987). En ella se describen 11 patrones funcionales y sus correspondientes criterios de valoración (CV), relacionados con la salud de las personas, familias y comunidades, que van desde la percepción/ control de la salud, hasta las creencias y valores. Son una configuración de comportamientos comunes, a mayor o menor medida, a todas las personas que intervienen en su salud, calidad de vida y capacidad de potencial humano. Aparecen de forma súbita, crónica o a largo plazo y permiten la valoración sistemática y ordenada del paciente a nivel bio- psico-social y de entorno.

\section{PLAN DE PROMOCIÓN DE LA SALUD}

Se define la promoción de la salud (PdIS) como la herramienta mediante la cual se otorga a la persona el poder y la capacidad de asumir mayor control sobre su propia vida y decidir sobre su salud, y así mejorar su calidad de vida.

Desde la conferencia de Alma Ata (1978) y la redacción de la carta de Otawa (1986), se refuerza el concepto de que mediante los planes de promoción de la salud se generan estrategias para aumentar el auto cuidado y así mejorar la calidad de vida de la población, ya que les da un papel activo en su propia salud y de manera indirecta se reducen los costes sanitarios.

La creación de un plan de promoción de la salud requiere de un abordaje multidisciplinar y holístico mediante el cual se cubran todos los aspectos a tratar. Es importante diferenciar los conceptos de promoción vs prevención, la PdIS va dirigida a mejorar la capacidad de vida desde un modelo de intervención en la que los individuos toman un papel de participación activa. En cambio la prevención en todos sus niveles parte del interés de disminuir enfermedades o factores de riesgo.

\section{El Precede.}

Modelo Precede, siglas en ingles de "Predispossing, reinforcing, and enabiling causes in educational diagnosis and evaluation" es un instrumento para el diagnóstico de las necesidades educativas del individuo o población, que permite la formulación de un plan de promoción de la salud para modificar conductas erróneas, abolir hábitos tóxicos o ampliar conocimientos que permitan una mejor calidad de vida. Diseñado por L. Green y M. Kreuter.

\section{Metodología del proyecto educativo}

Mediante el desarrollo del modelo PRECEDE, partiendo, no de una conducta errónea, sino de 
una conducta susceptible de un cambio para ampliar información, formación y actitudes firmes, es decir, sin experiencia ni conocimientos. La necesidad básica de la mejora de las experiencia sexual en verso una situación nueva. A través del proceso y mediante objetivos de aprendizaje divididos en ámbitos de habilidades, conocimientos y actitudes, se puede conseguir el cambio conductual esperado.

La parte activa del plan de promoción constará de 7 sesiones de 2 h de duración cada una. Se adaptara el horario a la mayoría del grupo y se estipulara un día entre semana a la misma hora, ej. Martes de 17 a $19 \mathrm{~h}$.

Por lo que el plan de promoción, una vez seleccionado el grupo, tendrá una duración de 6 semanas, $12 \mathrm{~h}$ en total. El elevado número de horas, y el concretar un día, permite establecer lazos de confianza entre el comunicador/ educador y el participante, que favorece la escucha activa y el feedback positivo. Elementos clave para que el mensaje sea claro y provoque un cambio de actitud, una adquisición de conocimientos y un desarrollo de las habilidades correctas.

Los grupos serán reducidos, de entre 4 y 6 mujeres.

La primera sesión será individual, con cada una de las participantes en las sesiones del plan de promoción de la salud en ella se valora el efecto de los factores predisponentes, facilitadores y reforzadores, imprescindibles para valorar la inclusión o no al proyecto.

Las otras 6 estarán orientadas a desarrollar las intervenciones dentro de las cuales se interrelacionan los objetivos de aprendizaje.

Los contenidos van delimitados en las intervenciones a realizar dentro de las sesiones.

Sesión 1: Presentaciones y definición de las necesidades del grupo.

Sesión 2: Introducción a la sexualidad: conceptos anatómicos, sexualidad vs salud sexual, limitaciones de la LM.

Sesión 3: Autoconcepto. Taller de autopercepción y fomento de la autoestima.

Sesión 4: Desmitificación de la sexualidad genital. La sexualidad sensorial, taller de autoexploración.

Sesión 5: Medidas técnicas en ergonomía y ortopedia sexual. Sesión para Parejas.

Sesión 6: Evaluación de las sesiones. Turno abierto de palabra para aportar ideas y sensaciones percibidas durante las sesiones.

La asistencia completa del taller se ha de realizar de manera presencial, donde todas las dinámicas estarán realizadas en grupo (4-6 mujeres) con puntuales actividades individuales, sesión 3 y 4.

En cuanto a los métodos utilizados serán el expositivo, el demostrativo mediante la técnica de role-playing, y el método de análisis además de discusiones haciendo uso de herramientas como el debate y la charla.

El planteamiento inicial en cuanto a método y actividades podrá ser modificado, en relación a las necesidades de las mujeres incluidas en el proyecto.

\section{Evaluación}

La evaluación de los objetivos se realizará por separado mediante una prueba de objetiva, demostraciones prácticas, asistencia y participación. Finalmente al finalizar las sesiones se les entregara un cuestionario de satisfacción adaptado, con ítems referentes a las presentaciones y sesiones, la utilidad de estas y las expectativas vs realidad, así como a la metodología empleada y la valoración del profesional de enfermería que ha impartido los cursos. 


\section{INTERVENCIONES ENFERMERAS EN CUIDADOS SANITARIOS DE SEXUALIDAD PARA MUJERES CON LESIÓN MEDULAR LUMBAR EN CATALUÑA}

\section{RESULTADOS Y CONCLUSIONES}

El proyecto se encuentra en fase de implementación, los resultados entran en proceso de análisis en la actualidad.

\section{BIBLIOGRAFIA}

Aguado A, Alcedo Mํㅡ A, García L. (2008). La sexualidad de las personas con lesión medular: aspectos psicológicos y sociales. Una revisión actualizada. Intervención psicosocial,Vol .17 ,n². p. 125-141.

ANSSYD y Sex Asistent, (2015). Código de Buenas Prácticas para Profesionales en Sexualidad y Diversidad Funcional. Barcelona: Disponible en: www.anssyd.es

Becker. E. (1978). Female sexuality following spinal cord injury. Accent Special Publications, Cheever Pub.

Bezerra, F., Taziana. S. (2013). Promoción de la salud: la calidad de vida en las prácticas de enfermería. Enferm. glob. [Internet]. Oct [citado el 27 de Marzo de 2016]; 12(32): 260-269. Disponible http://scielo.isciii.es/scielo.php?script=sci_arttext\&pid=S169561412013000400016\&ln g=es

BOE. Boletín Oficial del Estado. Orden SAS/1729/2010, de 17 de junio, por la que se aprueba y publica el programa formativo de la especialidad de Enfermería Familiar y Comunitaria. Sección III, p.57217.

BOE. (2011). Ley 26/2011, de 1 de agosto, de adaptación normativa a la Convención Internacional sobre los Derechos de las Personas con Discapacidad. n: 184: 87478- 87494)

Campo, M., Sánchez, A. La mujer con lesión medular: sexualidad y maternidad. Terapia sexual y de pareja. Vol 16; $p, 4-18$.

Carol,V.(1984):Pleasure and danger.Exploring female sexuality; Columbia, Routladge \& K.Paul.

Cobas C, Varela R.(1990). Manual de cuidados de enfermería para el lesionado medular y su familia. Xunta de Galicia.1990.

Curcoll, M.L, Vidal, J. (1992). Sexualidad y lesión medular. Blocs 3. Barcelona: Fundació Institut Guttman.

Espinaco, M.J., Ruiz, J.M., Román, 0. (1999). El personal de enfermería como educador en sexualidad. Una experiencia en Centros Docentes de Primaria y Secundaria. Sevilla.

Fallas MA, Artavia C, Gamboa A. (2012). Educación sexual: Orientadores y orientadoras desde el modelo biográfico y profesional. Revista Electrónica Educare,Vol 16. p. 53-71

Foro Vida Independiente.[Citado el 27 de marzo de 2016] Disponible en:www.forodevidaindependiente.org

Fuertes A, López F (1997). Aproximaciones al estudio de la sexualidad. Capítulo Sexto. Salamanca.

Generalitat de Catalunya.(1990) Departament de Benestar Social. Programa d'Actuacions Urgents per a Disminuïts. Lesió medul.lar. Que ens cal saber. ASPAYM. Catalunya;

Grado de Enfermería, Escuela de enfermería de Barcelona. (2015). Material docente, incluido en la asignatura Sexualidad y Reproducción Humana, de 4ํ. Barcelona: Universidad de Barcelona.

Grupo de Opinión del Observatorio de Bioética y Derecho. (2009). Análisis de los problemas bioéticos en personas con discapacidad..

IDESCAT. (1999). Estadistica de Discapacitats i Deficiencies.

INE. (2008) Encuesta de Discapacidad, Autonomía Personal y Situaciones de Dependencia.

Lowe, M. (1983): The dialectic of Biology and Culture, en Hubbard and Lowe, (eds.); Woman's Nature, NuevaYork, Pergamon Press

Marjory Gordon. Tipología de los patrones funcionales. Patrones funcionales de. Observatorio de metodología enfermera. [ Internet]. [citado el 26 de marzo de 2016]. Recuperado a partir de: 
http://www.ome.es/04_01_desa.cfm?id=391\#ancla7

NANDA, NIC, NOC, Metodología Enfermera. Actualización en Enfermería. [Internet].[Citado el 28 de marzo de 2016].Disponible en:http://enfermeriaactual.com/listado-criterios-noc/

Neil, M. R.; Watson, J. (2003) Filosofía y ciencia del cuidado. Modelos y teorías en enfermería, vol. 5, p. $145-64$.

Oliver S.S. (2011). Sexualidad, lesión medular y vida activa. Barcelona,

OMS. Centro de prensa Lesiones medulares. Datos y cifras. [Internet].[Citado el 27 de marzo de 2016]. Disponible en:http://www.who.int/mediacentre/factsheets/fs384/es/

ONU. (2006). Los Derechos y la dignidad de las personas con discapacidad. Convención sobre los Derechos de las personas con Discapacidad. [Internet]. [citado el 28 de marzo de 2016]. Disponible en :http://www.un.org/spanish/disabilities/default.asp?navid=12\&pid=497

Sánchez, E. (2015). Salud Sexual y calidad de vida en personas con traumatismo craneoencefálico moderado. Barcelona: Universitat de Barcelona.

Soler, A., Teixieira, T.C., Jaime, V.(2006). Discapacidad y Dependencia, Una perspectiva de Género. Madrid: Universidad Complutense de Madrid.

Tipología de las necesidades básicas. Necesidades Básicas de Virginia Henderson. Observatorio de metodología enfermera. [Internet ]. [ Citado el 27 de marzo de 2016]. Recuperado a partir de: http://www.ome.es/04_01_desa.cfm?id=424

UNICAN. Metodología de la EpS, Modelo Precede. Ciencias de la salud..[Internet].[Citado el 27 de marzo de 2016]. Disponible en:http://ocw.unican.es/ciencias-de-la-salud/promocion-de-lasalud/material-de- clase/metodologia-de-la-eps-i-modelo-precede 
